\title{
A young female with severe upper abdominal pain and profuse vomiting
}

\section{H. Hardardottir*, M.E. Keemers-Gels*, A. Termeer* and C. Rosman\#}

\section{CASE HISTORY}

A 23-yr-old female presented with progressive dyspnoea and epigastrical pain after 2 days of severe upper abdominal pain with heavy, profuse vomiting. There was no history of dysphagia or gastric reflux.

The patient was born with spina bifida, which was complicated with paralysis beneath L1, neurogenic bladder disease, hydrocephalus (for which a ventriculo-peritoneal shunt had been placed), and progressive thoracic scoliosis. Furthermore, she had a history of allergic bronchial asthma and had recently been treated with amoxycilline/clavulanicacid because of a urinary tract infection. Chronic medications included an oral contraconceptive and fluoxetine.

On physical examination the patient was in severe respiratory distress. She was cyanotic, very agitated and was lying in a strange position with her head between her paralysed legs, making physical examination very difficult. Furthermore, the patient's body temperature was $35^{\circ} \mathrm{C}$, with a blood pressure of $60 / 30 \mathrm{mmHg}$, a heart rate of 30 beats $\cdot \mathrm{min}^{-1}$ and no signal on the pulse oximeter. On pulmonary examination there were

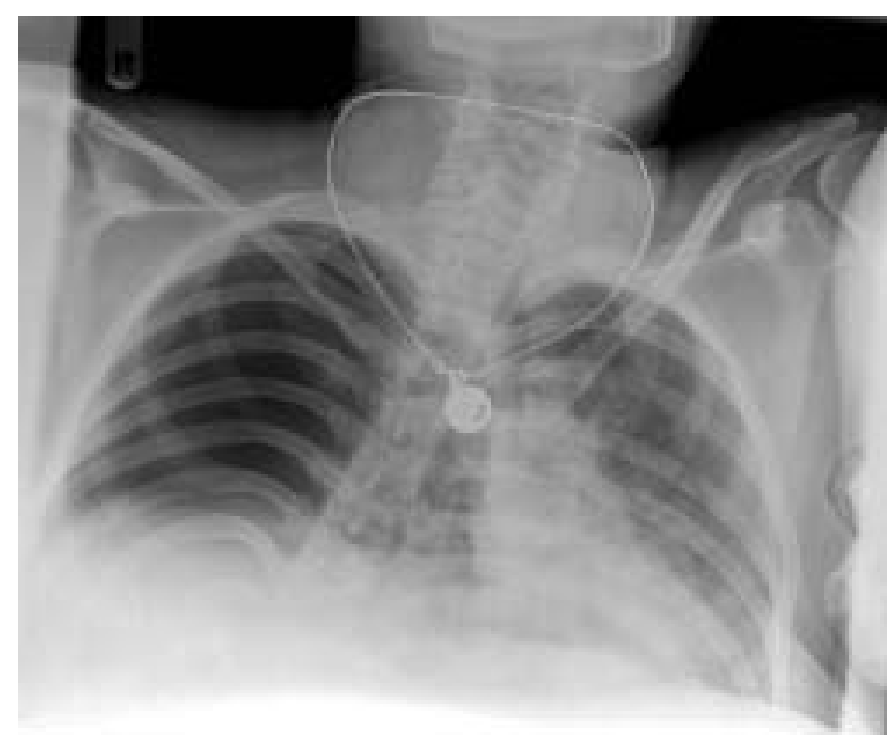

FIGURE 1. A chest radiograph at admission.

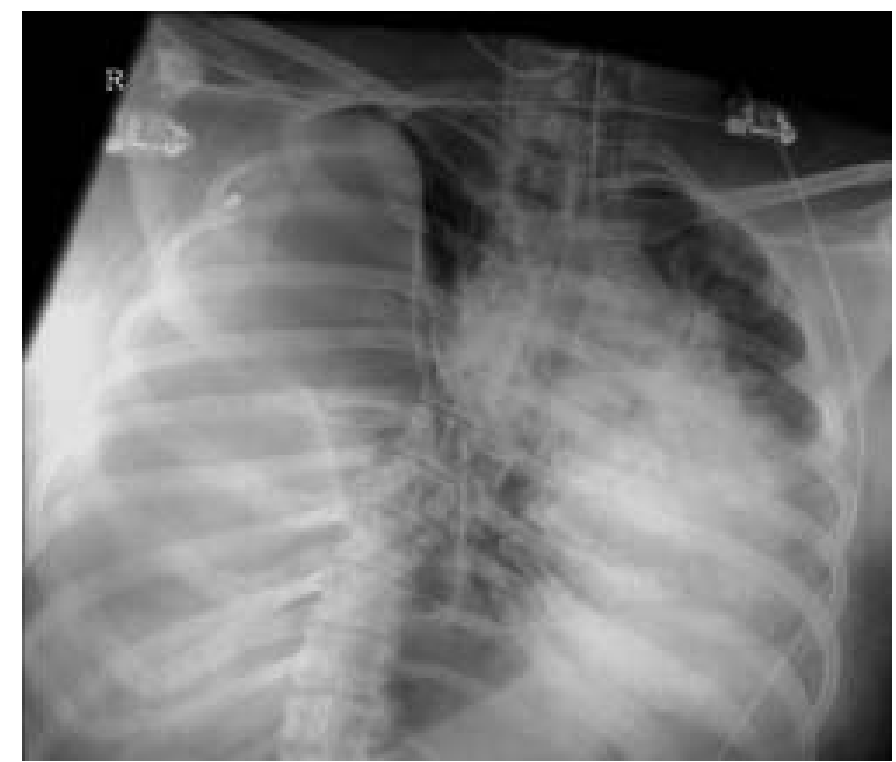

FIGURE 2. Posterioanterior chest radiograph after intubation and placing of a chest tube.

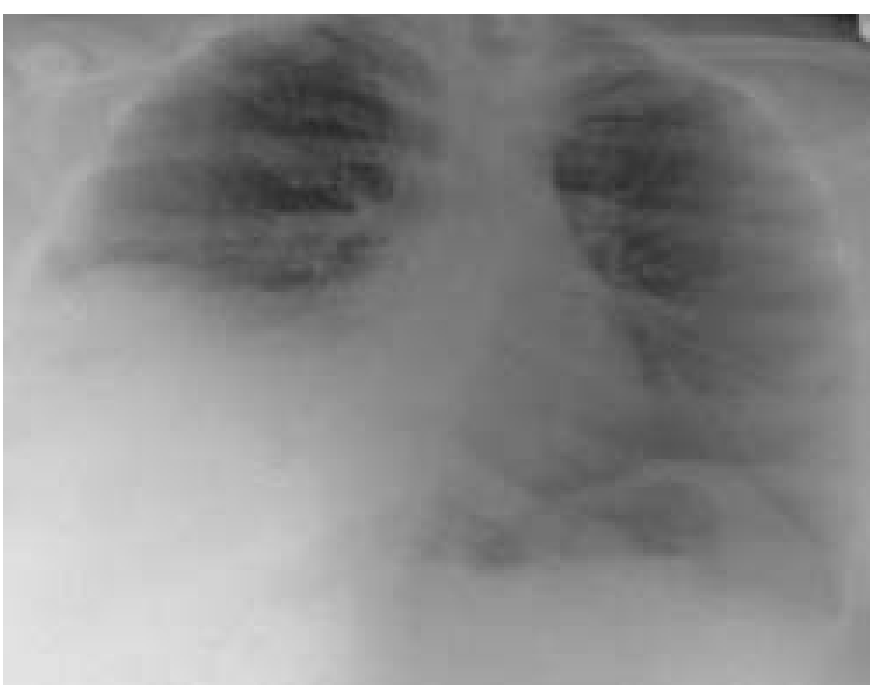

FIGURE 3. A chest radiograph of the patient taken 3 yrs previously.

Depts of *Pulmonology, and ${ }^{\#}$ Surgery, Canisius-Wilhelmina Hospital, Nijmegen, The Netherlands. CORRESPONDENCE: H. Hardardottir, University Medical Centre Nijmegen, Dept of Pulmonary Diseases (549), PO Box 9101, 6500 HB Nijmegen, The Netherlands. Fax: 31 243610324. E-mail: H.Hardardottir@LONG.UMCN.nl 
diminished breathing sounds with hypersonore percussion on the right side. On the left-hand side there were normal breathing sounds with rales of low frequency. Heart sounds were normal. Abdominal examination was not possible because of the position of the patient. A chest radiograph at admission is shown in figure 1 .

After evaluation of the chest radiograph the right hemithorax was punctured due to a strong suggestion of a tension pneumothorax. Under pressure, air escaped through the canule. This finding seemed to confirm the presumptive diagnosis of a right-sided tension pneumothorax. A chest tube was placed into the right hemithorax. However, the patient did not stabilise and had to be intubated and ventilated (fig. 2).

For comparison, a chest radiograph taken 3 yrs previously could be used (fig. 3).

\section{BEFORE TURNING THE PAGE INTERPRET THE CHEST RADIOGRAPHS AND SUGGEST A DIAGNOSIS.}




\section{INTERPRETATION}

The first chest radiograph demonstrated an aerated right hemithorax without vascular markings, mediastinal shift to the left-hand side with an unsharp left heart border.

The second chest radiograph revealed a striking difference, showing ground-glass appearance of nearly all of the right hemithorax, and a suggestion of a partially aerated lung at the top. On the left-hand side, perihilar consolidation was more pronounced than on the former radiograph, with unchanged mediastinal shift to the left.

The third chest radiograph, taken some years previously, shows an elevated right diaphragm and hiatus hernia extending alongside the heart and into the lung field on the righthand side.

\section{Diagnosis: Right-sided intrathoracic stomach and volvulus by sliding hiatal hernia.}

\section{CLINICAL COURSE}

After evaluation of the second chest radiograph and the clinical situation of the patient it was clear that the diagnosis was not a tension pneumothorax. Although the patient was now ventilated and treated with inotropics at the intensive care unit, the situation was still critical. The differential diagnosis was Boerhave syndrome because of the history with profuse vomiting. Other possibilities were discussed, but because of the pulmonary and haemodynamic instability, it was decided a thoracotomy should be performed.

During surgery, the entire stomach was found to be lying in the right hemithorax above the depressed right hemidiaphragm. The stomach was rotated, atonic, dilated and filled with fluid. The fundus showed necrosis. Part of the transverse colon with its omentum was also herniated into the right hemithorax. The thoracotomy was closed and a laparotomy was performed. The abdominal organs were moved into the abdominal cavity. Resection of the fundus and anterior gastropexy was performed.

There was a complicated post-operative course with adult respiratory distress syndrome and recurrent respiratory infections. The patient also needed prolonged ventilatory support. Eventually she was discharged for further rehabilitation.

\section{DISCUSSION}

Hiatal hernias represent a heterogenous clinical entity and may cause a variety of symptoms. This disease is seen in middleaged and older people mostly $>60$ yrs of age [1]. The patient had a right-sided intrathoracic stomach, which is a rare form of congenital sliding hiatal hernia $[2,3]$.

This abnormality is often discovered later in childhood because of the absence of severe complaints. The patient also had no history of dysphagia or gastric complaints.

Normally the cardia is anatomically situated at the level of the eleventh thoracic vertebra. In patients with a congenital sliding hiatal hernia the oesophagus is short, with the cardia situated at the Th7-Th9 vertebral level. This is at a significant cranial distance of the oesophageal hiatus. Therefore, the stomach migrates upwards and undergoes an anterior $180^{\circ}$ organoaxial torsion, with the greater curvature lying against the right chest wall. The gastrocolic ligament may pull a segment of the transverse colon into the thoracic cavity, as was found in the patient. During surgery the organs were moved into the abdominal cavity and gastropexia was performed after resection of the necrotic fundus [4].

The patient was in severe respiratory distress because of the mass effect with mediastinal shift. This was not due to a tension pneumothorax, but a distended stomach that fully filled the right hemithorax with tension on the mediastinum. The abdominal pain and vomiting the patient was suffering from in the preceding days can be explained by incarceration and strangulation of the stomach.

\section{REFERENCES}

1 Oddsdottir M. Paraesphageal hernia. Surg Clin N Am 2000; 80: 1243-1252.

2 Haddad M, Youssef B, Sammak B, Duff A. Right intrathoracic stomach secondary to congenital hiatal hernia and organoaxial torsion. Case reports. AJR Am J Roentgenol 1996; 167: 66-68.

3 Hubbert B, Toyama W. Familial right thoracic stomach. Paediatrics 1987; 79: 430-431.

4 Menguy R. Surgical management of large paraesophageal hernia with complete intrathoracic stomach. World J Surg 1988; 12: 415-422. 